\section{Population analysis}

Spatial Population Analysis. By P. H. Rees and A. G. Wilson. Pp. 356. (Arnold: London; Academic: New York, 1977.) £27.50; \$35.50.

ALL would agree with the authors' aim to provide good methods of population analysis for effective social and economic planning; this detailed and thorough text provides such an essential basis. In their preface, the authors warn the reader that he must face considerable conceptual difficulties in acquiring the skill, but comfort him by claiming relatively little demand on formal mathematical knowledge apart from algebra and an elementary knowledge of matrices. Nievertheless, this is a formidable text that needs to be painstakingly worked through to follow the argument.

The strength of the text is claimed to be that for the first time the analysis takes full cognisance of migration through the "use of new and powerful conceptual and mathematical tools". Such recognition of the importance of migration is welcome, since it has received scant treatment in some recently published work, a strange weakness in a world of growing mobility.

The text itself is arranged in five parts, each well introduced, and the argument subsequently explained and documented. The first part introduces current population models and their associated problems: here the authors introduce one of their fundamental features-models based on age groups and not life-tables, a basic divergence from the work of Keyfitz on the mathematics of population. It is also pointed out that although concentration is on theoretical concepts, examples using real data are introduced to illustrate the argument.

The second part introduces the concept of "population accounting" and is illustrated by reference to aggregate populations. The third part is devoted to the development of realistic population models, followed in the fourth part by the putting forward of the concept of life-table accounts to demonstrate that spatial dimensions may be added to conventional life-table analysis to make associated models more systematic, consistent and rigorous. The final, fifth, part summarises the new methods and their applications as well as their relationship to development in other fields.

The authors have been careful to make sure their work can be applied empirically by limiting themselves to commonly available data, although they have interwoven their own work with that of Lexis, Pressat, Rogers, Stone and others already accepted among demographers.

With page after page of mathematical formulae and matrices, the publishers have faced a monumental task in producing the book in present conditions at a realistic price. It is no wonder that the book is nevertheless expensive.

\section{R. E. H. Mellor}

R. E. H. Mellor is Professor of Geography at the University of Aberdeen, UK.

\section{$\gamma$-ray astronomy}

Gamma-Ray Astronomy. By E. L. Chupp. (Reidel: Dordrecht, The Netherlands and Boston, Massachusetts, 1976.) Cloth Dfl 105; paper Dfi 50.

THE title on the cover of this book reads Gamma-Ray Astronomy but inside a subtitle, Nuclear Transition Region has been added. This uncertainty as to the scope of the book exends into the text and gives an uneven quality to what is, otherwise, a valuable contribution to this field of astronomy.

The first chapter is a brief introduction and it is followed by a chapter on the fundamental mechanisms which produce $\gamma$ rays. Those processes which lead to line spectra, such as transitions between excited states of nuclei and the annihilation of positrons, are treated very thoroughly. Presumably for the sake of completeness this chapter also deals with mechanisms such as Compton scattering, bremsstrahlung and the decay of neutral pions, all of which lead to the production of continuous spectra.

The mechanisms discussed in chapter 2 are then used in the next chapter to predict the $\gamma$-ray emission which may be expected from astronomical sources. The inclusion of a chapter on predicted fluxes was perhaps necessary because of the paucity of experimental data in $\gamma$-ray astronomy, but this does lead to a certain amount of repetition in the book because some of the discussions are presented again when the experimental results are described in chapter 5. The author's interest in continuous spectra seems to waver a little in chapter 3 and he provides only a brief discussion of the high energy $\gamma$-ray emission from pulsars and supernova remnants, and makes no mention of extragalactic sources such as radio galaxies and quasars. Chapter 4 is concerned with the interactions of $\gamma$ rays with matter and is included as an introduction to experimental problems in $\gamma$-ray astronomy.

Chapter 5 is devoted to observational results. As in chapter 3, the treatment of the sources of continuous spectra is not consistent; for example, no mention is made of the many measurements of the pulsar NPO532 in the Crab nebula although the unsuccessful searches for $\gamma$ rays from extragalactic supernovae are described.

Chapter 6 deals with experimental problems in $\gamma$-ray astronomy and, appropriately, is the longest chapter in the book. It contains a comprehensive discussion of the background problems in a wide variety of detectors; if the quantity of data referred to does seem a little forbidding to the newcomer to the field, it will at least remind him of the need for technical innovation if this branch of astronomy is to make significant progress.

This is the first book to place the correct emphasis on the experimental problems in $\gamma$-ray astronomy and will therefore be invaluable both to the newcomer and to the experienced worker in this field. $\quad$ R. R. Hillier

R. R. Hillier is Lecturer in the Department of Physics, University of Bristol, UK.

\section{BOOKS \\ ON PURE \\ AND APPLIED SCIENCE}

\section{Books reviewed or men-}

tioned in this journal are available from stock.

Catalogues on application.

Please state interests.

\section{SCIENTIFIC LIBRARY}

ANNUAL SUBSCRIPTION from $\mathbf{f} 5.00$

Reduced rates for multiple subscriptions

Available in U.K. only

Prospectus free on request

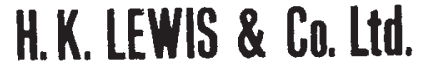

LONDON: 136 GOWER STREET, WC1E 6BS

Telephone : $01-3874282$ 